\title{
The cognitive profile of children treated with radiation for acute lymphoblastic leukaemia
}

\author{
S-J Whitaker, E Schutte \\ Department of Psychology, University of the Witwatersrand, Johannesburg \\ S-J Whitaker, BA Hons \\ E Schutte, MEd
}

Corresponding author: S-J Whitaker (sjdwhitaker@gmail.com)

\begin{abstract}
Background. Cranial radiation is part of a treatment protocol for childhood acute lymphoblastic leukaemia (ALL) in South Africa. Radiation is known to disrupt the myelination and integrity of white matter tracts in the brain. Associated cognitive impairment has been well documented in other countries, but not to the same extent in the multicultural and multilingual South African context.

Objectives. The current study focused on the assessment of memory and learning, two imperative cognitive functions. A quantitative evaluation of verbal and visuospatial memory performance in a cohort of ALL patients was done in order to establish whether there was a difference in performance between verbal and visuospatial tasks.

Methods. Eight patients with a low socio-economic background and being educated in their second language were included in the cognitive evaluation. All had received $18 \mathrm{~Gy}$ of radiation as part of their treatment protocol and were on maintenance treatment at the time of the study.

Results. In all the patients, primary cognitive impairment was demonstrated in ostensibly right hemisphere visuospatial tasks in comparison with ostensibly left hemisphere verbal tasks. Because deficits in visuospatial attention and working memory were identified, qualitative analysis of the results suggested that the white matter tracts in the right frontoparietal region and prefrontal cortex may be particularly vulnerable to radiation injury.

Conclusion. The study findings support vulnerability of the right hemisphere, particularly the right frontoparietal region and prefrontal cortex, to radiation injury. The decline in visuospatial cognitive abilities has major implications for second-language learners, as visuospatial learning is particularly important for them.
\end{abstract}

S Afr J CH 2012;6(4):128-131. DOI:10.7196/SAJCH.475

Demyelination induced by the radiation used in the treatment of childhood acute lymphoblastic leukaemia (ALL) diminishes the white matter in the brain. ${ }^{1}$ At least one neuropsychological consequence of demyelination is a reduction in processing speed. ${ }^{2} \mathrm{~A}$ brain region identified as being particularly sensitive to disruptions in white matter integrity is the prefrontal cortex, an area associated with functional working memory. ${ }^{3}$ These changes may adversely impact on neuropsychological functioning.

Structurally, the ratio of white to gray matter is significantly greater in the right than in the left cerebral hemisphere. ${ }^{4}$ In terms of hemispheric specialisation, speech and language functions are in most cases lateralised to the left hemisphere and visuospatial functions to the right. ${ }^{5}$ Given the uneven distribution of white matter in the brain and radiation-induced demyelination, we expected to see deficits in right hemisphere visuospatial memory tasks more than in left hemisphere verbal memory tasks.

Previous research findings have shown that children with ALL show evidence of slight deficits in verbal tasks but that primary impairment is evident in visuospatial tasks. ${ }^{6,7}$ Primary visuospatial impairment has also been reported in studies finding that ALL survivors showed reduced performance on visuospatial tests despite normal IQ scores. ${ }^{8}$ These findings suggest abnormalities in the white matter tracts of the right hemisphere.

\section{Objectives}

The current study evaluated the cognitive test performance of a cohort of children treated with a standard dosage of $18 \mathrm{~Gy}$ of cranial radiation for ALL. The questions under investigation were whether the cohort of patients showed differences in performance between verbal and visuospatial tasks, whether there was reduced performance in tasks involving a speed component, and whether they showed evidence of deficits in working memory.

\section{Methods \\ Sample}

The sample consisted of 8 patients who were attending a state hospital for the treatment of ALL at the time of the study. Informed consent was obtained from the guardians of the participants and minor assent was obtained from the participants. Participants were carefully selected on the basis of four inclusion criteria: ( $i$ ) having received 18 Gy of cranial radiation, which is administered for central nervous system prophylaxis in phase 3 of the treatment regimen -10 fractions of the $18 \mathrm{~Gy}$ dose are administered over a period of 10 consecutive days; (ii) in the maintenance phase of treatment (phase 5) at the time of the study; (iii) age between 7 and 18 years; and (iv) in the formal schooling system, with a minimum of 5 years of formal education. The age range of the sample was $11-17$ years and there was an even male/female split. The sample was homogeneous with regard to treatment protocol, length of treatment, second-language 
learning, attendance at government school and low socio-economic background. In addition, all the participants had previously attended crèche and preprimary school, indicating that they were on a normal development path prior to diagnosis of ALL. Since the test protocol was designed to include the English versions of the tests, patients who did not have a functional use of the English language were excluded, as were those with prediagnosed learning disabilities.

\section{Instruments}

Four psychometric tests were administered to assess different learning and memory abilities. The Rey Auditory Verbal Learning Test (RAVLT) and Digit Span subtest of the Senior South African Individual Scale - Revised (SSAIS-R) (a Wechsler-like test) were used to assess verbal learning and memory. The Rey-Osterrieth Complex Figure Test (ROCFT) and Coding subtest of the SSAIS-R were used to assess visuospatial learning and memory.

\section{Procedure}

Testing commenced with the administration of the first five learning trials of the RAVLT (RAVLT I-V) followed by presentation of a new list of words (RAVLT B) immediately thereafter to assess the effect of interference. The researchers noted the order of words recalled to assess the cognitive organisational strategy used by the participants in the verbal domain. Cognitive organisational strategy refers to the way new incoming auditory and visual information is categorised and stored in the brain. A 30-minute break was given before a delayed recall trial was administered to examine transfer to and retrieval from long-term memory. During the break, the copy trial of the ROCFT (ROCFT COPY) was administered. The researchers presented the participants with the figure to be copied and a set of eight coloured pencils that were provided sequentially by the researchers. The coloured pencils were used to assess the participants' cognitive organisational strategy in the visuospatial domain. Another 30-minute break was given before the final trial to assess delayed recall on the ROCFT was administered. During this break, the researchers administered the Coding subtest. The participants were given 120 seconds to copy the symbols, which allowed the researchers to assess speed of processing. Immediately after the copy trial, the participants were presented with an incidental associative memory task imposed on the original copy task. This step provided a measure of both free recall and visual-associative learning. A 30-minute break was given before the final trial to assess delayed recall of the Coding task was administered. During this break, the Digit Span subtest was administered. Both Digits Forward and Digits Backward trials were completed in this time. Following this, the final trials of each test (RAVLT DELAY, ROCFT DELAY) were administered to assess transfer to and retrieval from long-term memory. Finally, a word recognition trial (RAVLT REC) was administered to separate out factors of free recall and recognition.

\section{Results}

The raw scores obtained on each psychometric test were converted to standard $\mathrm{z}$-scores using the mean and standard deviation of a comparison sample, a group of asymptomatic learners demographically matched in terms of age, race, educational opportunity and socioeconomic status. The comparison sample was obtained from a current published South African study. ${ }^{9}$

\section{Cognitive profile}

Fig. 1 presents the mean $z$-score on each test obtained by the ALL group as a whole. With regard to the pattern across the first five learning trials of the RAVLT, the group started off exactly average, with a RAVLT I score of 0 , then peaked very quickly on the second trial and gradually dropped up to the fourth trial, with a final improvement on the last trial. Importantly, all scores on RAVLT I-V were in the average range when compared with the asymptomatic sample.

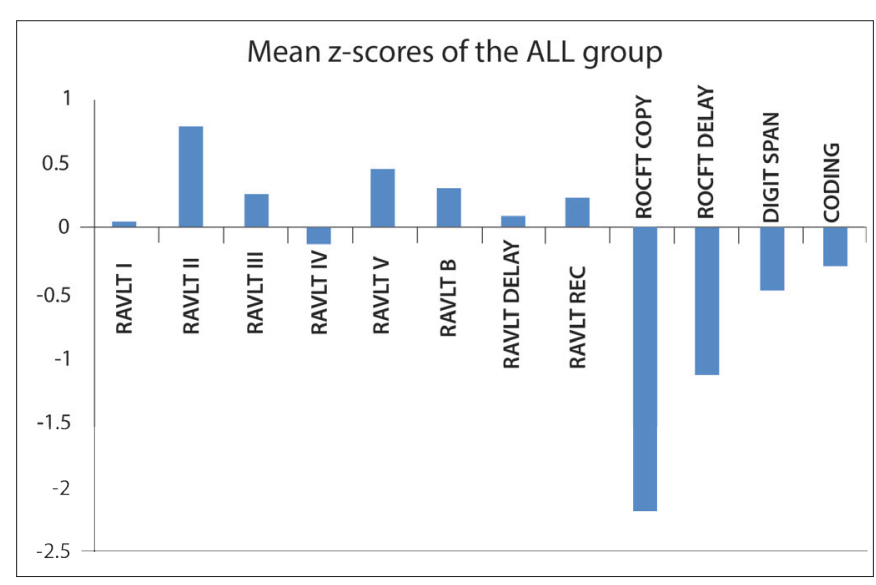

Fig. 1. Mean z-scores of the acute lymphoblastic leukaemia group.

For the delayed recall tasks, the $\mathrm{z}$-scores were in the average range for the verbal delay task (RAVLT DELAY) yet below average for the non-verbal delay task (ROCFT DELAY). The delayed recall trial on the RAVLT compared well with the asymptomatic group, whereas the delayed recall trial on the ROCFT was comparatively weaker.

A difference could be seen between overall verbal performance versus overall visuospatial performance of the group in that the mean $z$-scores obtained on the verbal tasks (RAVLT I-V, B, DELAY, REC) were generally positive, whereas the mean $\mathrm{z}$-scores obtained on the visuospatial tasks (ROCFT COPY, DELAY, CODING) were negative. However, all of the scores fell within the acceptable range, except for the ROCFT copy score and the ROCFT delayed recall score, which was possibly a factor of the low ROCFT copy score.

Overall performance on the Digit Span test was in the lower end of the average range; however, there was a notable difference in performance between the Digits Forward and Digits Backward spans of each participant.

The mean performance of the sample on the Coding task was within the acceptable range; however, an important observation made of all the participants was that they consistently referred back to the key above before filling in each code. When they completed an immediate incidental recall task, participants recalled an average of 6 codes out of 9 .

The final result was that performance on the RAVLT recognition task was only slightly superior to performance on the RAVLT delayed recall task.

With regard to the cognitive organisational strategy used in the verbal domain, the general pattern of recall was a serial order approach, whereby the participants recalled the words in the same randomised order in which the researchers had presented them. In the visuospatial domain, the general strategy used to copy the figure was a gestalt strategy, whereby the participants drew the outline of the figure first and then proceeded to fill in the smaller details.

\section{Discussion}

With regard to rate of learning, the sample was exactly average on RAVLT I, did their comparatively best performance on RAVLT II, and gradually lost their advantage up to RAVLT IV but somewhat recovered on RAVLT V. As validated by the Digit Span results, this was attributable to good attention and immediate memory span but weaker organisation and self-monitoring, until the participants eventually managed to organise the information in their heads. This suggests slow central processing in the cognitive organisation and consolidation of information. One important consideration is that for 
both the test and comparison groups, the RAVLT was administered in the language of schooling rather than the mother tongue. This may have implications for the overall validity of the test; however, the comparison group was carefully chosen so that cognitive profiles could be compared over like samples.

The ALL group appeared to be characterised by deficits particular to the ostensibly right hemisphere visuospatial domain, in view of the verbal versus non-verbal learning difference shown by the group. The weaker performance on the delayed recall trial of the ROCFT may be explained by the initial poor performance on the ROCFT copy trial. For example, some of the participants omitted certain design elements when copying the figure, which led to omission of those elements in the delayed recall trial. Others demonstrated inaccurate placement of design elements. This may relate to poor visual analysis and limited cognitive capacity. It suggests a deficit in visuospatial attention, which involves the right frontoparietal lobe. ${ }^{10}$ Previous imaging studies have related the decline in visuospatial ability to underlying white matter tracts in the frontoparietal region, which are vulnerable to radiation injury. ${ }^{11}$

The difference between overall verbal performance versus overall visuospatial performance suggested that the sample was relatively unimpaired in verbal tasks but comparatively weaker in non-verbal tasks. This finding demonstrated that memory differs according to the type of material being learned. It supported the hypothesis that right hemisphere visuospatial tasks would be more impaired that left hemisphere verbal tasks.

Although overall performance on the Digit Span test was in the average range, there was a marked difference between the Digits Forward and Digits Backward spans for each participant. The Forward and Backward spans for various age groups have been recorded in Table B.7 of the manual for the Wechsler Intelligence Scale for Children, 4 th edition (WISC-IV) ${ }_{12}^{12}$ which provides norms for the difference in span. In the current study, the differences in span obtained by the sample were greater than the norm differences in the WISCIV manual ${ }^{12}$ (p. 267). The difference in span demonstrates reliance on rote versus working memory: the relatively good performance on the Digits Forward part of the test was in line with the standard of rote verbal recall demonstrated on the RAVLT and supported the auditory attention established in the RAVLT. The greater difference in span demonstrates inefficient functioning of working memory and relates to the weaker organisation and self-monitoring of verbal output observed on the first four learning trials of the RAVLT. It highlighted the ability of the group to maintain information in working memory, yet they had difficulty reorganising the information and performing mental manipulations on it, as required by the Digits Backward task. Organisation and self-monitoring both involve the prefrontal cortex, which, in line with previous studies, suggests that this area may be particularly sensitive to disruptions in white matter integrity.

Although the processing speed of the sample was slightly negatively affected, it fell within the acceptable range. However, it was observed that the participants consistently referred back to the key throughout the task, which may have been due to visual memory deficits keeping processing on a more concrete level. When participants completed an immediate incidental recall task, which assessed visual-associative memory, no participant correctly recalled all the codes. This supported the theory that the processing of visual input occurred on a superficial rather than a deep level.

When the participants were given a word recognition task, it appeared that they were able to recognise the target words as familiar but had some difficulty distinguishing whether the target word was from list A or list B. This suggests that memory is not just of the item being stored but also of the context in which the memory was formed. Results reflected problems with source memory, which requires remembering the context in which something was experienced and is dependent on the prefrontal cortex. ${ }^{2}$ Owing to issues with source memory, recognition memory was only slightly superior to free recall in the ALL group.

In terms of the cognitive strategy used by the participants, the general pattern of subjective organisation was a serial order approach in respect of the verbal domain. This is an inefficient cognitive strategy compared with a semantic clustering strategy. A serial order strategy on the first five learning trials of the RAVLT may explain the average retrieval results in the delayed recall trial if words were not effectively placed into semantic categories. In addition, the tendency to repeat words already recalled on the same trial was evident on each trial for every participant. This tendency is known as perseverance and reflects attentional deficits in working memory and inconsistent self-monitoring. ${ }^{13}$ Imaging studies have related perseverance to reduced activity in the prefrontal cortex. ${ }^{14}$ Given that children who have been treated for ALL seem to be impaired in right hemisphere tasks, bimodal learning has been suggested to assist them - i.e. new material should be presented in both auditory and visual forms. ${ }^{15}$ The combination of visual and auditory cues may enhance the ability of ALL children to remember. Since the children in this study demonstrated deficits in the categorisation and organisation of new information in memory, a further suggestion would be to present new material to ALL children in a pre-organised way. This may help them to form an internal representation of the information in a way that is already organised and efficient.

The major limitation of this study was the small sample size; however, for purposes of validity we set very specific inclusion criteria. In addition, there was no pretest at diagnosis, so the study could not draw any causal conclusions. However, given the length of treatment for ALL and the difference in age between diagnosis and recovery, a pretest may be irrelevant. Finally, this study can only speculate about the brain regions involved in radiation injury, as no imaging techniques were used. However, all considerations were based on findings from previous brain imaging research.

Future studies should include a complete neuropsychological test battery in order to assess other cognitive domains such as attention and perhaps include a visuospatial learning test to assess the visuospatial learning curve, since only the verbal learning curve was assessed in this study. Inclusion of brain imaging techniques should also be considered. A further suggestion is to assess patients from a higher socio-economic background to determine the effect of socio-economic background and quality of education on test performance.

\section{Conclusion}

Although no causal conclusions can be drawn, the cognitive test results reveal primary visuospatial impairment following cranial radiation. These results may reflect underlying white matter abnormalities in the right cerebral hemisphere. The results also demonstrate the vulnerability of the prefrontal cortex and right frontoparietal region to radiation injury. Study findings are particularly relevant in the South African context where many children are second language learners: verbal ability in the second language is somewhat challenging, rendering visuospatial learning even more important. Finally, we have emphasised the value of bimodal learning and the presentation of structured material in assisting ALL survivors.

Funding, disclosures and conflicts of interest. This study had no specific funding. There are no financial disclosures from the authors, and no conflicts of interest.

Acknowledgements. The authors thank Dr B F Goodwin for his guidance in the study. 


\section{References}

1. Butler RW, Hill JM, Steinherz PG, Meyers PA, Finlay JL. Neuropsychologic effects of cranial irradiation, intrathecal methotrexate, and systemic methotrexate in childhood cancer. J Clin Oncol 1994;12(12):2621-2629.

2. Gazzaniga MS, Ivry RB, Mangun GR. Cognitive Neuroscience: The Biology of the Mind. 3rd ed. New York: WW Norton, 2009.

3. Nordahl CW, Ranganath C, Yonelinas AP, DeCarli C, Fletcher E, Jagust WJ. White matter changes compromise prefrontal cortex function in healthy elderly individuals. J Cogn Neurosci 2006;18(3):418-429. [http:// dx.doi.org/10.1162/jocn.2006.18.3.418]

4. Rourke BP. Nonverbal Learning Disabilities: The Syndrome and the Model. New York: Guilford, 1989.

5. Goldstein EB. Cognitive Psychology: Connecting Mind, Research, and Everyday Experience. 2nd ed. Belmont, CA: Thomson Wadsworth, 2008.

6. Copeland DR, Dowell RE, Fletcher JM, et al. Neuropsychological effects of childhood cancer treatment. J Child Neurol 1988;3:53-62. [http:// dx.doi.org/10.1177/088307388800300113]

7. Lesnik PG, Ciesielski KT, Hart BL, et al. Evidence for cerebellar-frontal subsystem changes in children treated with intrathecal therapy for leukemia: Enhanced data analysis using an effect size model. Arch Neurol 1998;55:1561-1568. [http://dx.doi.org/10.1001/archneur.55.12.1561]

8. Waber DP, Shapiro DL, Carpentieri SC, et al.Excellent therapeutic efficacy and minimal late neurotoxicity in children treated with 18 grays of cranial radiation therapy for high-risk acute lymphoblastic leukemia: A 7-year follow-up study of the Dana-Farber Cancer Institute consortium protocol 87-01. Cancer 2001;92(1),15-22. [http://dx.doi.org/10.1002/10970142(20010701)92:1<15::AID-CNCR1286>3.0.CO;2-W]

9. Skuy M, Schutte E, Fridjhon P, O'Carroll S. Suitability of published neuropsychological test norms for urban African secondary school students in South Africa. Personality and Individual Differences 2001;30:1413-1425. [http://dx.doi.org/10.1016/S01918869(00)00123-9]

10. Corbetta M. Frontoparietal cortical networks for directing attention and the eye to visual locations: Identical, independent, or overlapping neural systems? Proc Natl Acad Sci USA 1998;95:831-838. [http://dx.doi. org/10.1073/pnas.95.3.831]

11. Langleben DD, Segall GM. PET in differentiation of recurrent brain tumor from radiation injury. J Nucl Med 2000;41(11):1861-1867.

12. Wechsler D. Wechsler Intelligence Scale for Children. 4th ed. San Antonio, TX: Psychological Corporation, 2003.

13. Pollak Y, Kahana-Vax G, Hoofien D. Retrieval processes in adults with ADHD: A RAVLT study. Dev Neuropsychol 2008;33(1):62-73. [http:// dx.doi.org/10.1080/87565640701729789]

14. Hazlett EA, Buchsbaum MS, Jeu LA, et al. Hypofrontality in unmedicated schizophrenia patients studied with PET during performance of a serial verbal learning task. Schizophr Res 2000;43(1):33-46. [http://dx.doi. org/10.1016/S0920-9964(99)00178-4]

15. Jannoun L, Chessels MD. Long-term psychological effects of childhood leukemia and its treatment. Pediatr Hematol Oncol 1987;4:293-308. [http://dx.doi.org/10.3109/08880018709141282] 